\title{
Haematological validation of a computer-based bone marrow reporting system
}

\author{
D T Nguyen, L W Diamond, J D Cavenagh, R Parameswaran, J A Amess
}

\begin{abstract}
Aims-To prove the safety and effectiveness of "Professor Belmonte", a knowledge-based system for bone marrow reporting, a formal evaluation of the reports generated by the system was performed.

Methods-Three haematologists (a consultant, a senior registrar, and a junior registrar), none of whom were involved in the development of the software, compared the unedited reports generated by Professor Belmonte with the original bone marrow reports in 785 unselected cases. Each haematologist independently graded the quality of Belmonte's reports using one of four categories: (a) better than the original report (more informative, containing useful information missing in the original report); (b) equivalent to the original report; (c) satisfactory, but missing information that should have been included; and (d) unsatisfactory.

Results-The consultant graded 64 reports as more informative than the original, 687 as equivalent to the original, 32 as satisfactory, and two as unsatisfactory. The senior registrar considered 29 reports to be better than the original, 739 to be equivalent to the original, 15 to be satisfactory, and two to be unsatisfactory. The junior registrar found that 88 reports were better than the original, 681 were equivalent to the original, 14 were satisfactory, and two were unsatisfactory. Each judge found two different reports to be unsatisfactory according to their criteria. All 785 reports generated by the computer system received at least two scores of satisfactory or better.
\end{abstract}

Conclusions-In this representative study, Professor Belmonte generated bone marrow reports that proved to be as accurate as the original reports in a large university hospital. The haematology knowledge contained within the system, the reasoning process, and the function of the software are safe and effective for assisting haematologists in generating high quality bone marrow reports.

(f Clin Pathol 1997;50:375-378)

Keywords: knowledge-based systems; bone marrow reporting; laboratory management

Before healthcare providers can justify using a knowledge-based system or "decision support system" in preference to their usual methods of generating interpretive reports, the software must be judged to be accurate, safe, reliable, and effective. In addition, the system should allow the professional to utilise their own judgment when making decisions. ${ }^{1}$ To prove the quality of a medical knowledge-based system, formal validation studies must be carried out, preferably by reviewers who were not involved in the design of the system.

"Professor Belmonte" is a haematology knowledge-based system for bone marrow reporting. Belmonte is the third module in a comprehensive system for haematology laboratory diagnosis including "Professor Petrushka" for peripheral blood interpretation, and "Professor Fidelio" for flow cytometry immunophenotyping. Professors Petrushka and Fidelio are commercially available as part of the Coulter FACULTY Knowledge-Based System Software.

The three modules are designed to be used in concert on a haematology workstation or on several networked workstations. The modules share relational databases that act as a communication link between the programs. ${ }^{2}$ The structure and function of the three modules has been thoroughly described in the literature..$^{3-6}$

Professor Belmonte follows a logical approach to bone marrow diagnosis, classifying cases based on the patterns listed in table 1. As more than one pattern can coexist, a hierarchy has been established based on standard haematology principles. The "non-specific" pattern corresponds to the condition where no other pattern definition is met. If more than one pattern remains after the first evaluation of the data, an algorithm is applied to exclude all but the pattern with the highest ranking in the hierarchy. Each pattern corresponds to a list of differential diagnoses. The final diagnosis is established by using rules, specific to a given pattern, which take into consideration the clinical history, the peripheral blood pattern retrieved from Professor Petrushka's database, the morphological findings in the bone marrow, the results of cytochemical stains, and, when appropriate, the immunophenotyping data retrieved from Professor Fidelio's database. When the findings are non-diagnostic, Belmonte defaults to a descriptive diagnosis. Before printing the report, the contents of Belmonte's report can be edited, if desired, by the haematologist.

It is important to incorporate peripheral blood findings into the interpretation. Therefore, Belmonte automatically searches the peripheral blood database and displays the pertinent blood count and manual differential 
Table 1 Bone marrow patterns

Normocellular

Hypocellular

Erythroid predominance/hyperplasia

Hypercellular, myeloid predominance/hyperplasia

Megakaryocytic hyperplasia

Lymphocytosis/plasmacytosis

Mononuclear cell infiltration

Foreign cell infiltration

Granulomatous

Bi-hyperplasia

Non-specific

data at the beginning of the consultation. Based on the peripheral blood findings, specific suggestions are made for the bone marrow specimen, including reminders for appropriate adjunctive tests such as immunophenotyping and cytogenetics.

The specific morphological features found in the bone marrow aspirate and trephine are entered by the haematologist after microscopic review. The presence or absence of particles in the aspirate, an estimate of the cellularity, the relative number of megakaryocytes, and the presence of cells foreign to the bone marrow can be assessed at low power. Higher magnification is used for a bone marrow differential count and cytological evaluation of the erythroid and myeloid precursors.

If the bone marrow differential is judged to be normal, qualitative estimates of the relative numbers of erythroid and myeloid precursors can be entered instead of a detailed bone marrow differential. The characteristics (pattern and cytology) of any lymphoid infiltrate present on the trephine can be input. Choices are also available to indicate that no abnormal cells are present on the aspirate or trephine. When these selections are used, Belmonte appends the comment, "No evidence of infiltration", to appropriate sections of the report. Special stains, such as iron storage and incorporation, reticulin, and stains for myeloid and monocytic precursors, are entered when appropriate.

Professors Petrushka, Fidelio, and Belmonte have been installed on a five computer network in the haematology laboratory at St Bartholomew's Hospital in London, UK. Petrushka and Fidelio have been in live operation since January 16, 1996. Belmonte was added on June 17, 1996 after the computergenerated report was customised (fig 1) to closely resemble the reports previously issued by the department. The formal evaluation reported here was performed shortly after activating Professor Belmonte.

\section{Methods}

Cases with accession numbers from 1 to 800 in 1995 (January 2 to August 31) were chosen. During this time, 14 accession numbers were skipped because of clerical errors. Of the remaining 786 cases, one was excluded from the study because it was a postmortem trephine of poor quality.

For the 785 cases in the study, the demographic data (patient identification number, date of birth, and sex) and the clinical history obtained from the original bone marrow report were entered into the appropriate database using a Windows utility. ${ }^{7}$ The blood count and peripheral blood film findings (including the manual differential, if available) were collected from the laboratory computer system or from an archive on microfiche. The full blood counts were entered using the manual data entry utility supplied with Coulter FACULTY and processed with Professor Petrushka. In cases with flow cytometry immunophenotyping, the findings were processed with Professor Fidelio.

The microscopic findings (aspirate and/or trephine) contained in the original bone marrow reports were entered into Professor Belmonte. The 785 unedited reports generated by the system were printed and assembled into two binders (cases 1-400 and cases 401-800) analogous to the two bound volumes containing the laboratory's copies of the original reports.

Three haematologists, a consultant (JAA), a senior registrar (JDC), and a junior registrar (RP) compared Belmonte's report with the original report. None of the three was involved in the development of the software. Neither of the two registrars was responsible for any of the original reports.

The haematologists independently judged the quality of Belmonte's reports using four grades: "A+", "A", "S", and "U". The grade "A+" was given when Belmonte's report was judged to be better than the original (for example, when Belmonte's report contained useful information or a comment not included in the original). The grade of " $A$ " was assigned when the two reports were equivalent. An " $S$ " meant that Belmonte's report was satisfactory, but was either missing information that should have been included, or contained an unnecessary comment. When Belmonte's report was judged to be unsatisfactory, a grade of " $U$ " was assigned. Each reviewer had a separate score sheet with space available for comments. The haematologists were instructed to indicate their reasons for assigning the grade " $S$ " or "U". The results were tabulated from the score sheets.

In order to judge the overall quality of Belmonte's reports, a numerical score was assigned to each grade as follows: $\mathrm{A}+=1.25$, $A=1.00, S=0.70$, and $U=0$. A score for the overall quality of the reports was calculated for each judge. A mean score of 1.00 indicates that the overall quality of the reports is the same as that of the original reports.

\section{Results}

A summary of the grades assigned by the haematologists and the mean score for the overall quality of the reports is shown in table 2 . Between $86.7 \%$ and $94.1 \%$ of the reports were found to be equivalent to the original reports. Although each judge found two reports $(0.3 \%)$ to be unsatisfactory, there was no consistency in the faults recorded, and no single report received more than one unsatisfactory score. Thus, each of the 785 reports received a grade of satisfactory or better from at least two of the three haematologists. Depending on the judge, 
BONE MARROW REPORT

ST BARTHOLOMEW'S HOSPITAL, HAEMATOLOGY DIVISION

Patient's Name:

$$
\text { DOB : }
$$$$
22 / 8 / 1942
$$

Sex :

CR No.

Ward/Dept: Medical Oncology

Date Taken:

$30 / 1 / 1995$

Reason for marrow: Referral

Treatment:

No current FBC.

SITE (S) :

CELLULARITY :

Increased.

MEGAKARYOCYTES: Present in normal numbers.

ERYTHROPOIESIS: Reduced; Normoblastic.

GRANULOPOIESIS: Reduced cellularity.

LYMPHOID CELLS: LYmphocytes account for $80 \%$ of nucleated cells. ABNORMAL CELLS :

IRON STAIN :

OTHER STAINS:

TREPHINE :

Hypercellular marrow with normal numbers of megakaryocytes.

The bone marrow is infiltrated by small

lymphoid cells in a diffuse pattern.

INTERPRETATION: Diffuse involvement by a lymphoproliferative disorder.

COMMENT :

The differential diagnosis includes: CLL/SLL, and low-grade NHL.

The following tests may be helpful to

subclassify the LPD: Haemogram and

blood film, FCM immunophenotyping,

a lymph node biopsy if lymphadenopathy is

present, and serum and/or urine protein

electrophoresis to look for a

monoclonal spike.

Figure 1 An unedited bone marrow report generated by Professor Belmonte. The patient information has been deleted. This report received a grade of " $A+$ " from two judges and " $A$ " from the third judge. FBC, full blood count; CLL/SLL, chronic lymphocytic leukaemia/small lymphocytic lymphoma; NHL, non-Hodgkin's lymphoma; LPD, lymphoproliferative disorder; FCM, flow cytometry.

Table 2 Quality of Professor Belmonte's reports

\begin{tabular}{llllll}
\hline fudge & $A+$ & $A$ & $S$ & $U$ & Mean score \\
\hline $\begin{array}{l}\text { Consultant } \\
\begin{array}{l}\text { Senior } \\
\text { registrar }\end{array}\end{array}$ & $64(8.1 \%)$ & $687(87.5 \%)$ & $32(4.1 \%)$ & $2(0.3 \%)$ & 1.01 \\
$\begin{array}{l}\text { Junior } \\
\text { registrar }\end{array}$ & $88(11.2 \%)$ & $681(86.7 \%)$ & $14(1.8 \%)$ & $2(0.3 \%)$ & 1.02 \\
\hline
\end{tabular}

from $1.8 \%$ to $4.1 \%$ of the reports received only a satisfactory grade. A variety of different kinds of minor faults were documented. The most consistent reason for assigning a score of satisfactory was a problem with a comment about iron status, often in patients with a normal mean cell volume. In some cases, the reviewers felt that a comment about iron status should have been made but Belmonte failed to do so, and, in some cases, the reviewers questioned the appropriateness of a comment regarding iron status that Belmonte appended to the report. Between $3.7 \%$ and $11.2 \%$ of Belmonte's reports received the A+ grade, mostly because either Belmonte's wording of the diagnosis was

clearer and more informative than the original diagnosis; or as the system was more consistent in integrating the peripheral blood pattern with the bone marrow findings, Belmonte mentioned something significant that was omitted in the original report. The mean numerical score for overall quality ranged from 1.00 to 1.02 , indicating that each of the haematologists judged Belmonte's unedited reports to be of the same quality as the original reports.

All three haematologists assigned the same grade to Belmonte's reports in 633 of the 785 cases $(80.6 \%)$. There was agreement by two of the three judges in 144 cases $(18.4 \%)$. No agreement was reached in only eight cases $(1.0 \%)$. If the grades of $\mathrm{A}+$ and $\mathrm{A}$ are taken together-so that two scores of $A$ and one of $\mathrm{A}+$ or two scores of A+ and one of A are considered agreement - the complete concordance rate between the three judges would be $93.4 \%$ (733 of 785 reports).

\section{Discussion}

Before a medical knowledge-based system can be accepted by healthcare providers for making decisions and generating interpretive reports, proper testing of the software is necessary to ascertain its safety and effectiveness. Although proving that the software will perform as specified under all conditions is inherently difficult, evaluation of a sufficiently large number of unselected cases, including difficult cases, should be sufficient to assure that the knowledge, data structures, algorithms, and inference methods are valid. Furthermore, this kind of formal evaluation, with adequate amounts of unbiased, representative test data, should give an accurate measure of the overall quality of the system's interpretations.

In a preliminary evaluation of the Professor Belmonte system for bone marrow reporting, 100 consecutive cases of leukaemia and malignant lymphoma were studied, and the results compared with the original diagnoses and the opinions of the developers. ${ }^{5}$ Professor Belmonte's reports agreed with the original diagnosis in $94 \%$ of cases and with the opinion of the developers in $99 \%$ of cases. Although this previous study was useful in establishing the quality of Belmonte's diagnoses in haematopoietic malignancies, the cases studied did not encompass all aspects of bone marrow pathology, the number of cases was relatively small, and the use of the developers as judges did not eliminate all bias in measuring the performance of the system.

In this study, the evaluation was on a large number of unselected cases, involving three haematologists with different levels of experience, none of whom had participated in the development of the software. The original bone marrow data, clinical history, peripheral blood findings, and where appropriate, immunophenotype, were entered into the computer system. The cases represented eight months of workload in a university hospital where between 1000 and 1200 bone marrows are examined annually. Approximately $40 \%$ of the cases were aspirates only and $60 \%$ had trephine biopsies. The cases were representative of all of 
the pathology encountered in the university setting including evaluation of unexplained anaemia, new patients presenting with acute and chronic leukaemias, and staging or follow up of patients with a variety of malignancies. The evaluation included both routine cases as well as difficult cases.

Each of the 785 reports generated by Professor Belmonte earned a score of satisfactory, or better, from at least two of the three judges. As the system correlated the peripheral blood findings with the bone marrow findings assiduously, and generated diagnoses with consistent wordings, the judges considered a substantial number of Belmonte's reports (3.7-11.2\%) to be better than the original reports. In addition, the mean calculated scores for overall quality showed that each judge found Professor Belmonte's reports to be as accurate as the original reports.

Although several groups have reported using knowledge-based systems and computer networks to improve the efficiency of reporting full blood counts, ${ }^{8-10}$ few laboratories have applied this technology to bone marrow reporting. Smith and Gyde developed two systems aimed at improving bone marrow reporting. ${ }^{11}{ }^{12}$ The first is a prototype rule-based expert system using a multiparameter approach for the diagnosis of leukaemia. The program, which incorporates clinical data including general laboratory tests, immunophenotype, morphology, and cytogenetics, was evaluated on 43 cases, demonstrating an agreement between the system diagnosis and the true diagnosis in $82 \%$ of cases. ${ }^{11}$ Although their system was designed only for leukaemia diagnosis, its multiparameter approach is similar to Professor Belmonte. Belmonte is only one module in a comprehensive system for diagnostic haematology which includes Professor Petrushka for peripheral blood interpretation, and Professor Fidelio for flow cytometry immunophenotyping (both commercially available in the Coulter FACULTY Knowledge-based Systems). Our systems are designed to correlate all available information including the clinical history and laboratory data related to haematology. The second program by Smith and Gyde is a rule-based bone marrow reporting system for generating the descriptive portion of the bone marrow report via a series of questions which the user answers by selecting choices from menus. ${ }^{12}$ Unlike Professor Belmonte, however, this system does not provide an interpretation of any kind.

The efficiency of a knowledge-based system is an important feature in addition to safety and accuracy. Comparison of the turnaround time of reports typed by secretaries to that of reports generated by Professor Belmonte demonstrated that the system nearly halved the bone marrow reporting time at St Bartholomew's
Hospital (from a mean of 10.08 days to 5.34 days for all reports), eliminating the previously existing four to five day delay caused by the backlog of reports and the limited availability of secretaries. ${ }^{13}$ The system is now routinely used for issuing bone marrow reports in the laboratory. If desired, the haematologist can edit the computer-generated report prior to printing. Currently, the main delay in issuing bone marrow reports is the expected time for batch-processing trephine specimens in the histopathology department. Up to $60 \%$ of the aspirates are now reported either on the day of the procedure or within one day. In the near future, it will be possible to interface Professor Belmonte to the hospital patient management system for electronic transmission of the complete reports to the appropriate wards, further speeding the communication of laboratory results. Origins of the unusual names of the three knowledge-based systems-
Professors Petrushka, Fidelio, and Belmonte are named after a professors Petrushka, Fidelio, and Belmonte are named after a actual names come from the world of classical music. Petrushka (a ballet by Stravinsky) was assigned to the peripheral blood interpretation module because the parrot is very colourful, like the hues in a Romanovsky stained peripheral blood film. Immunophenotyping by flow cytometry (in a liquid medium) is the domain of Fidelio (Beethoven's only opera) because a walrus spends much of his life in water and his brown colour reminds one of the nickname ("brown stain") used by pathologists for immunohistochemistry. The bone marrow module is the responsibility of Belmonte (a principal character in Mozart's responsibility of Belmonte (a principal character in Mozart's opera, The Abduction from the Seraglio) because the shape of the
bottlenose dolphin, in particular his rostrum, is reminiscent of a bottlenose dolphin, in

1 Brahams D, Wyatt J. Decision-aids and the law. Lance 1989;ii:632-4.

2 Diamond LW, Nguyen DT. Communication between exper systems in haematology. In: Richards B, ed. Healthcare com puting 1993: current perspectives in healthcare computing. Weybridge, UK: BHJC Books, 1993:111-19.

3 Nguyen DT, Diamond LW, Priolet G, Sultan C. Expert system design in hematology diagnosis. Methods Inf Med 1992;31:82-9.

4 Diamond LW, Nguyen DT, Andreeff M, Maise RL, Braylan RC. A knowledge-based system for the interpretation of flow cytometry data in leukemias and lymphomas. Cytometry 1994;17:266-73.

5 Diamond LW, Mishka VG, Seal AH, Nguyen DT. Multiparameter interpretive reporting in diagnostic laboratory hematology. Int $\mathcal{F}$ Biomed Comput 1994;37:21 1-24.

6 Nguyen D, Diamond L. Expert systems for laboratory hematology. L'Eurobiologiste 1993;27:323-30.

7 Diamond LW, Mishka VG, Seal AH, Nguyen DT. A clinica database as a component of a diagnostic hematology workstation. In: Osbolt JG, ed. Proceedings of the symposium on computer applications in medical care. $\Im$ Am Med Informatics Assoc 1994;1 (suppl):298-302.

8 Burgess PR, Kershaw GW, Coleman RH, Robin H, Coad $\mathrm{CD}$, Gibson J, et al. A computerized expert system for handling the output of the Technicon H1 hematology anahandling the output of the Technicon H1
lyser. Clin Lab Haemat 1993;15:2-321.

9 Arnott AJ, Copson P. A network-based laboratory data processing system for use with the Coulter ${ }^{\circledR}$ STKS haematology analyser. Clin Lab Haemat 1994;16:65-74.

10 Tolmie CJ, du Plessis JP. An expert system for the interpretation of full blood counts and blood smears in hematology laboratory. Artif Intell Med 1991;3:271-85.

11 Smith AG, Gyde OHB. Leukaemia diagnosis-a challenging problem for an expert system. In: Lun KC, Degoulet $P$ North-Holland, 1992:596-601.

12 Smith AG, Gyde OHB. Computer-assisted bone marrow reporting - a novel use for an expert system. In: Lun KC,
Degoulet $\mathrm{P}$, Piemme TE, Rienhoff O, eds. MEDINFO 92. Amsterdam: North-Holland, 1992:602.

13 Nguyen DT, Diamond LW, Cavenagh JD, Morris E, Parameswaran R, Amess JA. Increased efficiency of bone Parameswaran R, Amess JA. Increased efficiency of bone marrow reporting with hematology
tems. Lab Hematol 1997;3:10-18. 